\title{
Aproveitamento do resíduo agroindustrial de abacaxi submetido ao processo de secagem para elaboração de barra de cereais
}

\author{
Utilization of pineapple agroindustrial residue submitted to the drying process for the elaboration
} of cereal bars

Utilización de residuos agroindustriales de piña sometidos al proceso de secado para la preparación de barras de cereales

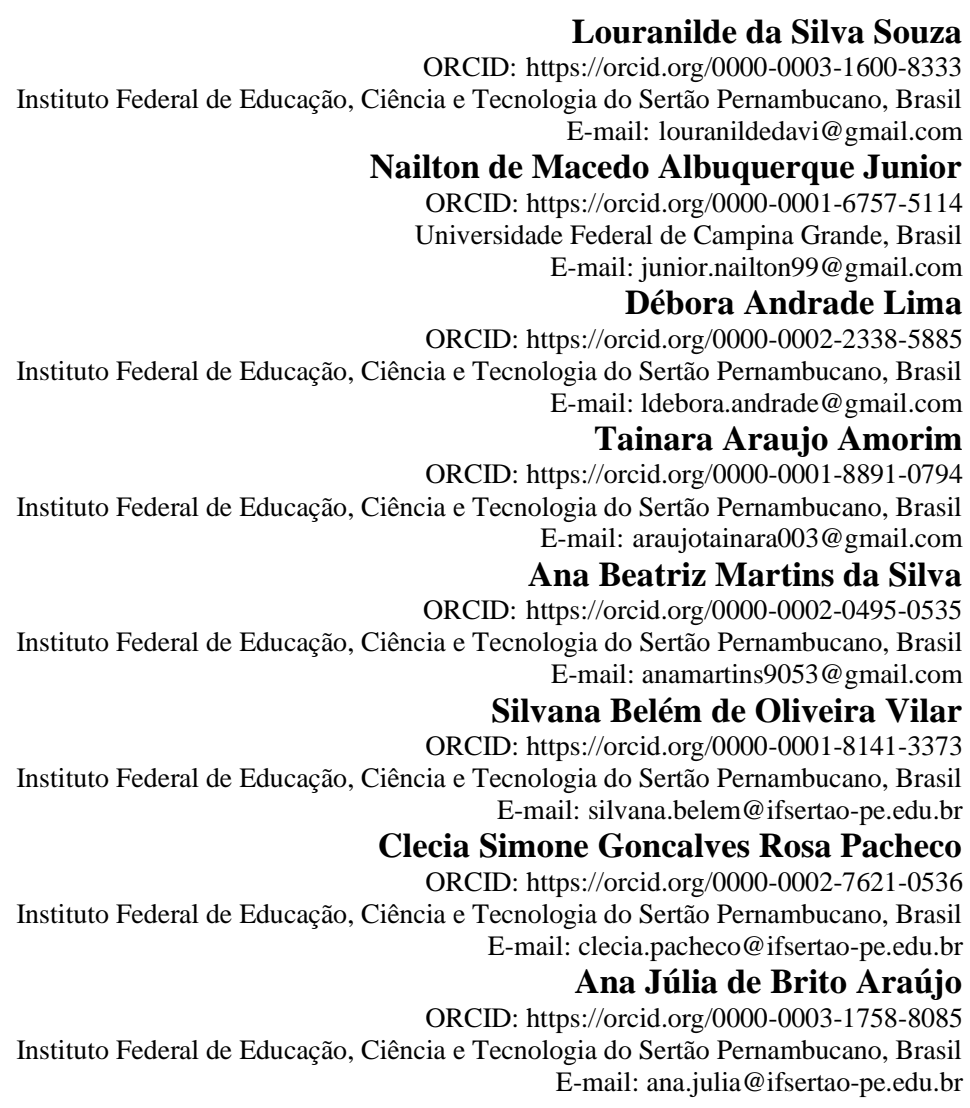

\begin{abstract}
Resumo
O abacaxi é uma fruta bastante cultivada mundialmente, no entanto o seu processamento agroindustrial gera resíduos os quais não apresentam destino adequado. O presente trabalho teve como objetivo estudar o aproveitamento do resíduo agroindustrial do abacaxi submetido ao processo de secagem e posterior adição em barra de cereais. Para obtenção do pó, o resíduo foi submetido ao processo de secagem convectiva a $60^{\circ} \mathrm{C}$, realizando-se a cinética de secagem e ajuste dos modelos matemáticos aos dados experimentais. Verifica-se que todos os modelos se ajustarem bem aos dados experimentais, sendo o modelo de Page considerado o melhor para representar o processo de secagem. Após a adição do pó nas formulações das barras de cereais verifica-se que a formulação B3 com $4,5 \%$ de pó do resíduo agroindustrial de abacaxi apresentou resultados satisfatórios. Quanto a textura, para o parâmetro de firmeza não foi observado diferença significativamente ao nível de 5\% nas formulações padrão e com adição do pó do resíduo agroindustrial de abacaxi e a avaliação da cor indica tendência para o vermelho e amarelo. Quanto a quantificação dos compostos o pó apresentou maiores concentrações, sendo a catequina o principal composto. Sendo assim é possível verificar a eficiência da adição do pó do resíduo de abacaxi no enriquecimento das barras de cereais em relação a sua composição de compostos bioativos e atividade antioxidante, promovendo a obtenção de uma barra de cereal com propriedades funcionais.
\end{abstract}

Palavras-chave: Nutrientes; Formulações; Compostos bioativos; Propriedades funcionais. 


\begin{abstract}
The pineapple is a fruit widely cultivated in the world, however its industriais processing generates waste, where these still contain relevant nutrients. The present work aimed to study the use of pineapple residue submitted to the drying process to obtain powder and develop cereal bars. To obtain the powder the residue was submitted to the convective drying process, performing the drying kinetics and the adjustment of mathematical models to the experimental data. It was verified that all models fitted well to the experimental data, with Page's model being considered the best to represent the drying process. After the addition of the powder in the formulations of cereal bars it was verified that a higher concentration in relation to the standard bar, being that the formulation B3 with $4.5 \%$ of powder of the pineapple agroindustrial residue was the best, while the others did not differ. As for the instrumental firmness parameter, the applied force did not differ significantly at the 5\% level in the standard formulations and with the addition of pineapple agroindustrial residue powder, and all the colorimetric results obtained were positive, indicating a tendency of color more towards red and yellow. As for the quantification of the compounds, the powder presented higher concentrations, catechin being the main compound. Thus, it is possible to verify the efficiency of the addition of pineapple residue powder in the enrichment of cereal bars in relation to its composition of bioactive compounds and antioxidant activity, promoting the obtaining of a cereal bar with functional properties.
\end{abstract}

Keywords Nutrients; Formulations; Bioactive compounds; Functional properties.

\title{
Resumen
}

La piña es una fruta ampliamente cultivada en el mundo, sin embargo su procesamiento industrial genera residuos, donde estos aún contienen nutrientes relevantes. El presente trabajo tuvo como objetivo estudiar el aprovechamiento del residuo de piña sometido al proceso de secado para obtener el polvo y desarrollar barras de cereal. Para obtener el polvo se sometió el residuo al proceso de secado convectivo, realizando la cinética de secado y el ajuste de los modelos matemáticos a los datos experimentales. Se comprobó que todos los modelos se ajustaban bien a los datos experimentales, siendo el modelo de Page el que se consideró mejor para representar el proceso de secado. Después de la adición del polvo en las formulaciones de barras de cereales se verificó que una mayor concentración en relación a la barra estándar, siendo que la formulación B3 con 4,5\% de polvo del residuo agroindustrial de piña fue la mejor, mientras que las demás no se diferenciaron. En cuanto al parámetro de firmeza instrumental, la fuerza aplicada no difirió significativamente al $5 \%$ en las formulaciones estándar y con la adición de polvo de residuo agroindustrial de piña, y todos los resultados colorimétricos obtenidos fueron positivos, indicando una tendencia de color más hacia el rojo y el amarillo. En cuanto a la cuantificación de los compuestos, el polvo mostró mayores concentraciones, siendo la catequina el principal compuesto. De esta manera se puede comprobar la eficacia de la adición de polvo de residuo de piña en el enriquecimiento de barras de cereales en relación a su composición de compuestos bioactivos y actividad antioxidante, promoviendo la obtención de una barra de cereales con propiedades funcionales.

Palabras clave: Nutrientes; Formulaciones; Compuestos bioactivos; Propiedades funcionales.

\section{Introdução}

A produção de frutas no Brasil, por meio do cultivo de diversas espécies, está alcançando a cada dia, um maior desempenho na agricultura nacional, com cerca de 2 milhões de hectares do território nacional ocupado, ocasionando na geração de mais de 5 milhões de empregos no campo, fazendo com que o país continue como o terceiro maior produtor de frutas do mundo por ano, com uma produção estimada em 44 milhões de toneladas, permanecendo atrás apenas da China e da Índia (Ibge, 2016).

Devido à grande quantidade de frutas, muitas indústrias têm às utilizado para elaboração de subprodutos como sucos e polpas, com intuito de elaborar produtos de fácil consumo. No entanto, o processamento de frutas acarreta a geração de resíduos, causando assim impactos ambientais quando não descartados de forma correta. Estima-se que cerca de $40 \%$ da fruta se transforme em resíduo, composto principalmente de casca, sementes e bagaço (Bortolotti et al., 2013; Nogueira et al., 2019).

O abacaxi é uma fruta tropical que contém alta concentração de açúcares, vitaminas e minerais e é produzida em larga escala no Brasil. O uso industrial da fruta é destinado à produção de suco e polpa, gerando uma grande quantidade de resíduos . Curiosamente, esses resíduos tornam-se um subproduto, pois além da presença enzimática da bromelaína, também pode atuar como um composto antimicrobiano e antioxidante (Oliveira, 2018).

Dessa forma, para muitos pesquisadores o estudo de alternativas que altere o destino para dos resíduos que em sua grande maioria são descartados é o foco principal, sendo uma das maneiras principais é a partir da elaboração de farinhas de frutas e vegetais e a aplicação em produtos alimentícios de diversas áreas, principalmente na indústria de panificação, sempre 
visando o enriquecimento nutricional de formulações alimentícias comerciais. Essas formulações alternativas vêm sendo avaliadas quanto à sua qualidade nutricional e aceitação sensorial (Menon et al, 2014; Karovicová et al., 2013; Ade et al, 2014).

A produção de farinha através do processo de secagem torna-se a alternativa ao uso de resíduos industriais, pois é um componente importante, rico em fibras, compostos bioativos, minerais, vitaminas e antioxidantes e tem vários impactos positivos à saúde (Marques, 2013; Brizola, 2014). O desenvolvimento de farinha permite a produção de novos produtos que podem aumentar as propriedades sensoriais, especialmente sabor e aumentar os nutrientes que são cada vez mais necessários no mercado para seus numerosos benefícios para a saúde (Macagnan et al., 2014).

Diante a importância do aproveitamento dos resíduos em relação as questões ambientais, e o aumento do valor nutricional de formulações de alimentos o presente estudo tem como objetivo obter a farinha do resíduo de abacaxi e desenvolver barras de cereais, avaliando as características físico-químicas e bioativas, de forma a minimizar o despejo do material e obter um produto de maior valor agregado.

\section{Metodologia}

O presente trabalho foi realizado no Instituto Federal de Educação, Ciência e Tecnologia do Sertão Pernambucano, Campus Petrolina-PE, no Laboratório Experimental de Alimentos (LEA). A abordagem metodológica foi determinada de forma quantitativa, recolhendo dados por meio das medições de grandezas, produzindo dados que podem ser avaliados através de métodos matemáticos como porcentagens, estatísticas e probabilidades, além de métodos numéricos, analíticos e o desenvolvimento de equações e/ou fórmulas matemáticas aplicáveis a algum processo (Pereira et al., 2018).

\subsection{Preparo da Matéria-prima}

O resíduo agroindustrial do abacaxi utilizado nesse trabalho foi cedido por uma indústria de beneficiamento de frutas, localizada na Cidade em Petrolina-PE. O mesmo foi transportado para o Laboratório Experimental de Alimentos (LEA). O aproveitamento do resíduo a ser estudado nesse trabalho será feito com o resíduo desidratado conforme apresentado na Figura 1 .

Figura 1. Resíduo de abacaxi in natura e desidratado.
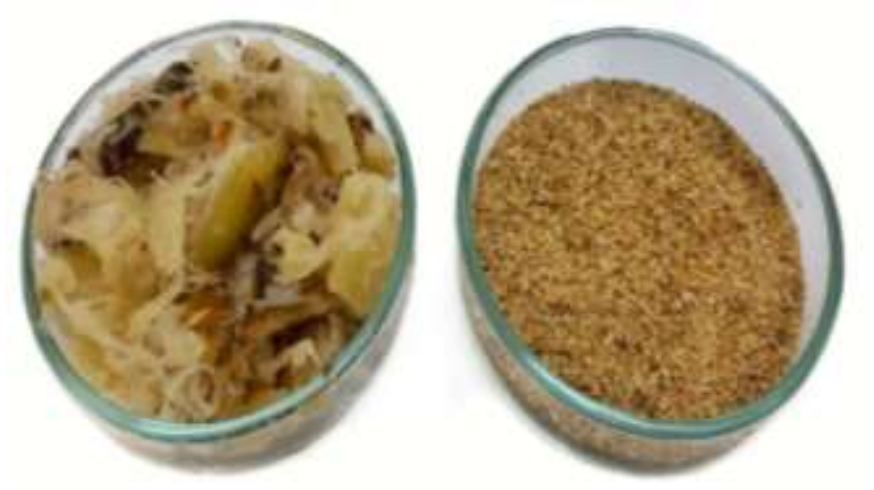

Fonte: Autores (2021).

\subsection{Secagem Convectiva}

A secagem do resíduo agroindustrial de abacaxi (RAA) foi realizada em um secador de bandejas com circulação forçada de ar ajustado para operar na temperatura de $60^{\circ} \mathrm{C}$. A temperatura de secagem foi escolhida com base nas faixas usualmente utilizadas para produtos agrícolas. Os experimentos foram realizados em triplicata, sendo as pesagens do resíduo feitas em balança analítica até atingirem peso constante. 
Após o processo de secagem, o resíduo será submetido à moagem em um moinho de facas, em seguida padronizados quanto a granulometria em 600 mesh e por fim embalados a vácuo em embalagens de polietileno de alta densidade sendo devidamente identificado.

\subsection{Cinética de Secagem}

As curvas de secagem foram obtidas pela conversão dos dados referentes à perda de água no parâmetro adimensional razão de teor de água $(\mathrm{RX})$, para as diferentes condições de secagens, mediante a Equação 1.

$$
R X=\frac{X_{b s}-X_{e}}{X_{b s \text { incial }}-X_{e}}
$$

onde:

RX = Razão de umidade (Adimensional) num tempo t;

$\mathrm{Xe}=$ Teor de água de equilíbrio em base seca $(\%)$;

$\mathrm{Xbs}=$ Teor de água em base seca num tempo $\mathrm{t}(\%)$;

Xbs $($ inicial $)=$ Teor de água inicial em base seca $(\%)$.

\subsection{Modelos Matemáticos}

Os valores experimentais para a razão de umidade ao longo do tempo e para cada temperatura do ar de secagem, foram preditos por modelos empíricos demonstrados na Tabela 1.

Para análise dos ajustes dos modelos matemáticos aos dados experimentais, foi utilizado o programa computacional Statistica versão 12.0, utilizando-se a análise de regressão não-linear, pelo método Quasi-Newton. Os modelos foram selecionados tomando-se como parâmetro a magnitude do coeficiente de determinação $\left(\mathrm{R}^{2}\right)$.

Tabela 1. Modelos Matemáticos aplicados para ajuste dos dados experimentais.

\begin{tabular}{cc}
\hline Modelos & Equação \\
\hline Henderson \& Pabis & $\mathrm{RX}=\operatorname{aexp}(-\mathrm{kt})$ \\
\hline Page & $\mathrm{RX}=\exp \left(-\mathrm{kt}^{\mathrm{n}}\right)$ \\
\hline Logarítmico & $\mathrm{RX}=\mathrm{aexp}(-\mathrm{kt})+\mathrm{c}$ \\
\hline Lewis & $\mathrm{RX}=\exp (-\mathrm{kt})$ \\
\hline
\end{tabular}

Fonte: Autores (2021).

Os critérios usados para determinação do melhor ajuste dos modelos aos dados experimentais foram o coeficiente de determinação $\left(\mathrm{R}^{2}\right)$ e do erro quadrático médio calculado pela Equação 2.

$$
D Q M=\sqrt{\frac{\sum\left(R X_{e x p}^{*}-R X_{p r e}^{*}\right)^{2}}{N}}
$$

onde:

N = Número de Termos;

RXpre = Razão de umidade predita pelo modelo;

RXexp = Razão de umidade experimental. 


\subsection{Elaboração das Barras de Cereais (Formulações)}

As matérias-primas para produção das barras foram obtidas em mercado local, sendo estas utilizados na produção: melaço, aveia, flocos, passas, castanha do Pará e castanha de caju.

Uma formulação padrão da barra foi adotada, e a partir dela foram elaboradas três formulações com diferentes proporções da farinha de resíduo do abacaxi (FRA), sendo alterado a proporção da aveia em cada formulação conforme a Tabela 2 abaixo.

Tabela 2. Proporções dos ingredientes para produção das barras de cereais.

\begin{tabular}{ccccc}
\hline Ingredientes & Barra Padrão & Barra 1 (2,5\%) & Barra 2 (3,5) & Barra 3 (4,5\%) \\
\hline Melaço & $87 \mathrm{~g}$ & $87 \mathrm{~g}$ & $87 \mathrm{~g}$ & $87 \mathrm{~g}$ \\
\hline Aveia & $52 \mathrm{~g}$ & $46,60 \mathrm{~g}$ & $44,46 \mathrm{~g}$ & $42,30 \mathrm{~g}$ \\
\hline Flocos & $29,5 \mathrm{~g}$ & $29,5 \mathrm{~g}$ & $29,5 \mathrm{~g}$ & $29,5 \mathrm{~g}$ \\
\hline Passas & $20 \mathrm{~g}$ & $20 \mathrm{~g}$ & $20 \mathrm{~g}$ & $20 \mathrm{~g}$ \\
\hline Castanha do Pará & $13,5 \mathrm{~g}$ & $13,5 \mathrm{~g}$ & $13,5 \mathrm{~g}$ & $13,5 \mathrm{~g}$ \\
\hline Castanha de Caju & $13,5 \mathrm{~g}$ & $13,5 \mathrm{~g}$ & $13,5 \mathrm{~g}$ & $13,5 \mathrm{~g}$ \\
\hline FRA & $0 \mathrm{~g}$ & $5,39 \mathrm{~g}$ & $7,54 \mathrm{~g}$ & $9,70 \mathrm{~g}$ \\
\hline
\end{tabular}

Fonte: Autores (2021).

\subsection{Caracterização Físico-Química}

O resíduo de abacaxi in natura e o pó foram caracterizadas em triplicata, avaliados quanto aos parâmetros físicoquímicos: teor de umidade, determinado pelo método de secagem das amostras em estufa a $105{ }^{\circ} \mathrm{C}$ até peso constante, sólidos solúveis totais (SST) a partir da leitura do índice de refração no refratômetro $\mathrm{ABBE}, \mathrm{pH}$ determinado pelo método potenciométrico utilizando em pHmetro marca Tecnopon (Modelo mPA - 210P/Versão 7.1), cinzas e acidez total titulável (ATT) seguindo os procedimentos propostos pela metodologia do Instituto Adolfo Lutz (Brasil, 2008). Para a determinação da firmeza foi utilizado o texturômetro TA. XT (Stable Micro Systems, UK), a análise de cor foi determinada por leitura direta utilizandose colorímetro HunterLab XE Plus, modelo 4500 L, com sistema de cor Cielab, sendo as coordenadas cromáticas L* que representa a luminosidade, transição do branco (0) para o preto (100), a* que representa a transição da cor verde (-a*) para a cor vermelha $\left(+a^{*}\right)$ e b* a transição da cor azul (-b*) para a cor amarela $\left(+b^{*}\right)$.

\subsection{Caracterização dos compostos bioativos}

\subsubsection{Preparo do Extrato}

Para a realização das análises referentes a capacidade antioxidante, conteúdo bioativo total e perfil de fenólicos e perfil de açúcares e ácidos orgânicos por cromatografia líquida de alta eficiência (CLAE), os extratos foram obtidos utilizando a proporção de 10 gramas do resíduo agroindustrial do abacaxí para $100 \mathrm{~mL}$ de solvente (etanol 50\%), realizadas em banho de ultrassom com contato indireto (Ultranique Q5. 9/40 A - com frequência de $40 \mathrm{kHz}$, potência de $132 \mathrm{~W}$ ).

\subsubsection{Determinação do conteúdo bioativo total (CBT)}

Foi determinado através do método espectrofotométrico com o reagente Folin-Ciocalteu, seguindo a metodologia descrita por Singleton e Rossi (1965) adaptada por Lima et al. (2014). Os resultados de absorbâncias obtidas foram comparados com a curva de calibração e expressos em mg EAG/g de Fenólicos Totais equivalentes ao ácido gálico. 


\subsubsection{Determinação da capacidade antioxidante}

A capacidade antioxidante foi determinada pelo método de captura do radical livre 2,2-difenil-1-picrilhidrazila (DPPH) de acordo com a metodologia de Kim et al. (2002) adaptada por Lima et al. (2014). O percentual de inibição do radical DPPH foi obtido conforme a equação 3.

Equação 3. Equação percentual de inibição do radical DPPH.

$$
\mathrm{PI}_{\mathrm{DPPH}}=\left[1-\left(\frac{A B S \text { amostra }_{\mathrm{DPPH}}}{\text { ABS } \text { controle }_{\mathrm{DPPH}}}\right)\right] \times 100
$$

Sendo:

$\mathrm{PI}_{\mathrm{DPPH}}=$ Percentual de Inibição do radical DPPH;

ABS amostra DPPH = absorbância da solução de DPPH adicionada da amostra e incubada no escuro por 30 minutos;

ABS controle ${ }_{\mathrm{DPPH}}=$ absorbância do controle sem adição de amostra no tempo 0 .

A determinação pelo método FRAP foi realizada segundo Rufino et al., (2006), com algumas adaptações. A absorbância foi medida em $595 \mathrm{~nm}$ no espectrofotômetro e os resultados obtidos comparados com a curva padrão de sulfato ferroso nas concentrações de 100 - $2000 \mu$ mol L-1, e expressos em mmol de Fe 2+ por litro da amostra.

\subsubsection{Perfil de compostos fenólicos}

Determinado por cromatografia líquida de alta eficiência (CLAE), utilizando um sistema HPLC Agilent modelo 1260 Infinity LC (Agilent Technologies, Santa Clara, CA, USA) equipado com bomba quaternária de solventes (modelo G1311C), degaseificador, compartimento de colunas termostatizado (modelo G1316A) e amostrador automático (modelo G1329B). Acoplado em Detector de Arranjos de Diodos (DAD) (modelo G1315D). Os dados obtidos foram processados utilizando o software OpenLAB CDS ChemStation EditionTM (Agilent Technologies), utilizando o procedimento analítico conforme descrito pela metodologia de Padilha et al. (2017).

\subsection{Análise Estatística}

Os resultados obtidos foram analisados em triplicata por meio do delineamento inteiramente casualizado, sendo submetidos à análise de variância (ANOVA), teste de comparação de médias de Tukey ao nível de $5 \%$ de significância, com o auxílio do programa SPSS version 20.0 for Windows (IBM, USA).

\section{Resultados e Discussões}

\subsection{Caracterização físico-química do resíduo agroindustrial do abacaxi in natura}

Na Tabela 3 estão expressos as médias e os desvios padrões referentes a caracterização físico-química do resíduo de abacaxi in natura. 
Tabela 3. Caracterização físico-química do resíduo de abacaxi in natura.

\begin{tabular}{cc}
\hline PARÂMETROS & RESULTADOS \\
\hline Umidade $(\%)$ & $79,66 \pm 0,17$ \\
\hline $\mathrm{pH}$ & $3,93 \pm 0,15$ \\
\hline Acidez Titulável Total (\%) & $1,84 \pm 0,01$ \\
\hline Sólidos Solúveis ( ${ }^{\circ}$ Brix) & $9,93 \pm 0,51$ \\
\hline Cinzas $(\%)$ & $4,70 \pm 0,10$ \\
\hline
\end{tabular}

Fonte: Autores (2021).

De acordo com Morzelle et al. (2015) os teores de umidade dos frutos sofrem influência por fatores como região de plantio, qualidades do solo, clima, época de colheita e grau de maturidade do fruto, que também podem interferir nas características físicas e químicas da fruta. Dessa forma, observou-se que a umidade apresentou um elevado teor de 79,66 \% devido a sua grande quantidade de água presente no resíduo, sendo este mais suscetível ao desenvolvimento de microrganismos, diminuindo assim o seu período de conservação e demonstrando a sua alta perecibilidade.

O resíduo apresentou um pH correspondente a 3,93, valor este próximo ao encontrado por Lima (2019) com um valor de 3,78, podendo ser caracterizados como um alimento ácido, estando seguro do ponto de vista microbiológico. Segundo Vilar et al. (2020) o potencial hidrogeniônico é um parâmetro que depende de alguns fatores como o estado de conservação e condições das matérias-primas. $\mathrm{O}$ valor encontrado $1,84 \pm 0,01 \%$ indicaram uma baixa acidez indicando uma susceptibilidade ao desenvolvimento de microrganismo. De acordo com Moreno (2016), a acidez titulável total representa todos os grupos de ácidos (ácidos orgânicos, livres, na forma de sais e compostos fenólicos) presentes em determinada amostra.

Os resultados obtidos na análise de sólidos solúveis totais (Brix) mostram que o resíduo apresenta teores de açúcares significativos $(9,93 \pm 0,51)$, podendo estar relacionado ao estágio de maturação que fruta se encontrava, pois quanto mais avançado o estádio de maturação da fruta mais elevados serão os teores de sólidos solúveis totais.

O teor de cinzas obtido no presente estudo foi de 4,70 $\pm 0,10 \%$, Monteiro et al. (2014), encontraram valores de cinzas nas amostras dos resíduos de abacaxi de $0,64 \mathrm{~g}\left(100 \mathrm{~g}^{-1}\right)$, valor este inferior ao encontrado no presente estudo, podendo estar relacionado da localidade onde a variedade foi plantada e da composição do solo onde se desenvolveu (Bortolatto \& Lora, 2008), as cinzas em alimentos referem-se ao resíduo inorgânico remanescente da queima da matéria orgânica, sem resíduo de carvão (Nunes et al., 2017).

\subsection{Modelagem Matemática}

Os parâmetros obtidos a partir dos ajustes dos modelos de Midili, Henderson \& Pabis, Page, Logarítmico e Lewis aos dados experimentais das cinéticas de secagem do resíduo agroindustrial do abacaxi encontram-se descritos na Tabela 4, bem como o coeficiente de determinação $\left(\mathrm{R}^{2}\right)$, erro médio quadrático (DQM). 
Tabela 4. Ajuste aos modelos de Midili, Henderson \& Pabis, Page, Logarítmico e Lewis.

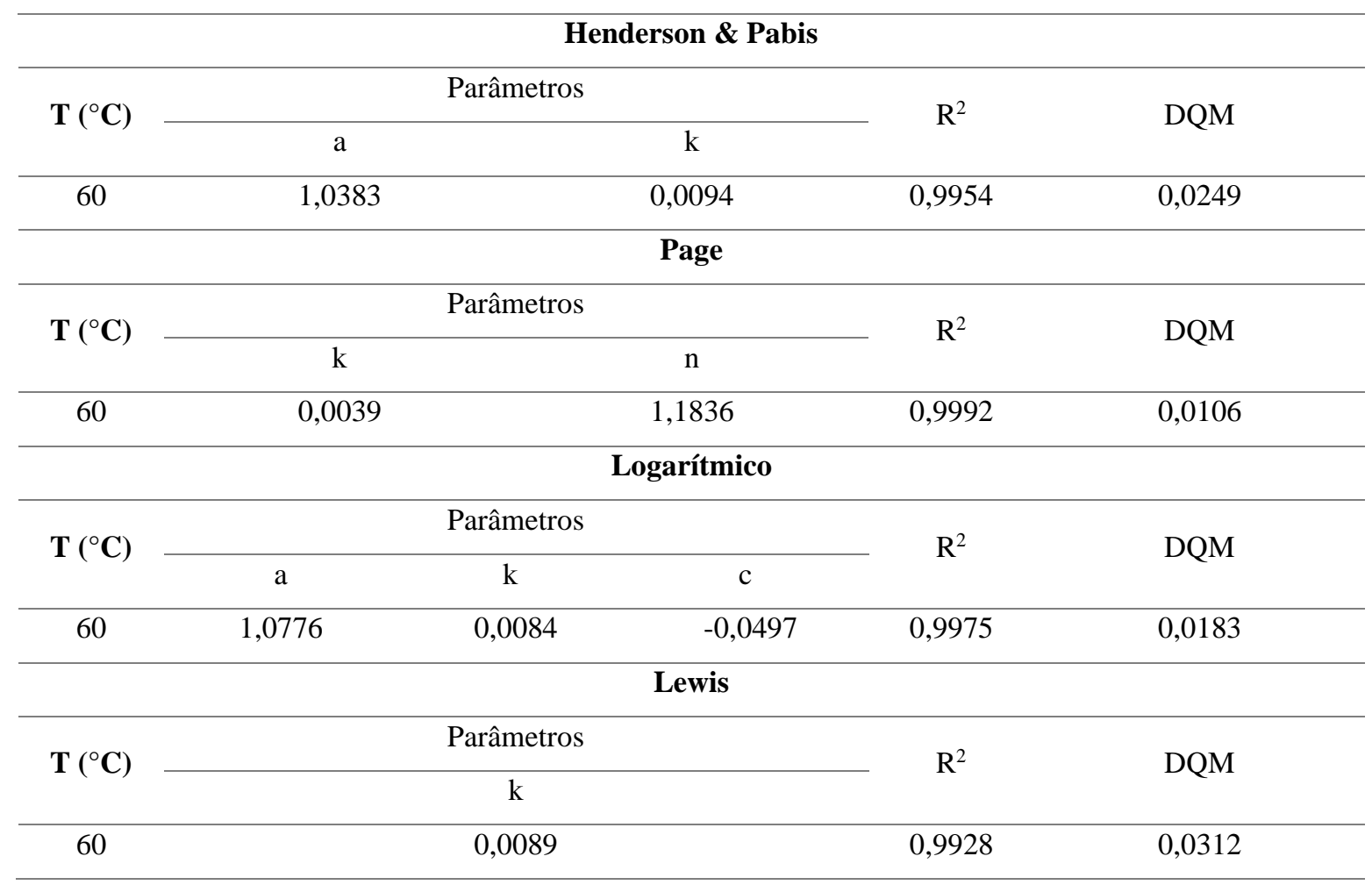

Fonte: Autores (2021).

Segundo Moscon et al. (2017) o coeficiente de determinação $\left(\mathrm{R}^{2}\right)$ varia entre 0 e 1 , indicando o quão próximo está entre os dados obtidos e os estimados. Verifica-se na tabela 4 acima que os modelos matemáticos ajustados aos dados experimentais apresentaram valores para coeficientes de determinação $\left(R^{2}\right)$ iguais ou superiores a 0,99 na temperatura do ar de secagem aplicada $\left(60^{\circ} \mathrm{C}\right)$ observando que todos os modelos aplicados representam adequadamente o processo de secagem estudado.

De acordo com Martins et al. (2015) somente o $\mathrm{R}^{2}$ não constitui um bom critério de seleção de modelos não-lineares quando avaliados de forma isolada. Com isso ao avaliar o erro quadrático médio (DQM), verificou-se que o menor valor foi para o modelo de Page quando comparados aos outros modelos avaliados no presente estudo, tornando-se o mais eficaz para descrever o processo de secagem do resíduo agroindustrial do abacaxi. Contudo, deve-se ressaltar que os modelos de Henderson e Pabis, Logarítmico e Lewis também apresentaram altos valores de $\mathrm{R}^{2}$ e baixos valores de DQM, indicando adequação para também representar a cinética de secagem.

\subsection{Cinética de Secagem}

Após a obtenção dos dados experimentais a partir da secagem, verificou-se que a temperatura do ar de secagem utilizado no processo teve influência nas curvas de secagem, pois quanto maior a temperatura mais rápido se obtém a umidade de equilíbrio constante, que é atingida quando o material perde totalmente o seu teor de água, fato corroborado por Kaveh et al. (2017), devido ao fato de que a umidade da superfície evapora muito rapidamente devido aos altos coeficientes de transferência de calor e massa nos processos de secagem em camada fina, salientando que a taxa de secagem reduz exponencialmente quando toda a umidade da superfície evapora e a secagem se difunde para dentro do material.. Leva-se em consideração que a temperatura de $60{ }^{\circ} \mathrm{C}$ utilizada no trabalho é aplicada para produtos agrícolas devido ser intermediária e não agredir tanto o teor nutricional.

Ao analisar os parâmetros todos os modelos matemáticos analisados podem ser utilizados para representar o processo de secagem do resíduo agroindustrial de abacaxi devido aos elevados coeficientes de determinação $\left(\mathrm{R}^{2}\right)$ e baixos valores do erro quadrático médio (DQM), o que favorece no aumento do nível de confiança dos ajustes. 
As curvas de secagem do resíduo agroindustrial do abacaxi obtidas na temperatura $60^{\circ} \mathrm{C}$ estão apresentados nas figuras abaixo, representadas pela razão de umidade em função do tempo para todos os modelos matemáticos ajustados.

Figura 2. Ajuste do modelo de Henderson \& Pabis aos dados experimentais da secagem do resíduo de abacaxi.

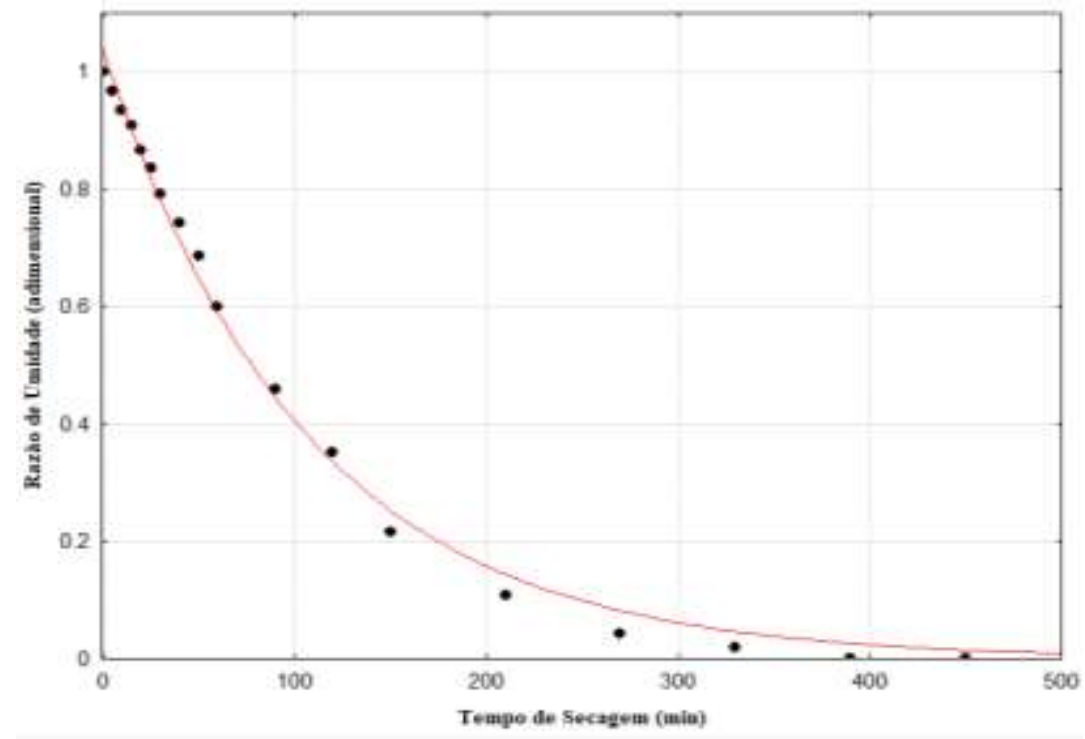

Fonte: Autores (2021).

Figura 3. Ajuste do modelo de Page aos dados experimentais da secagem do resíduo de abacaxi.

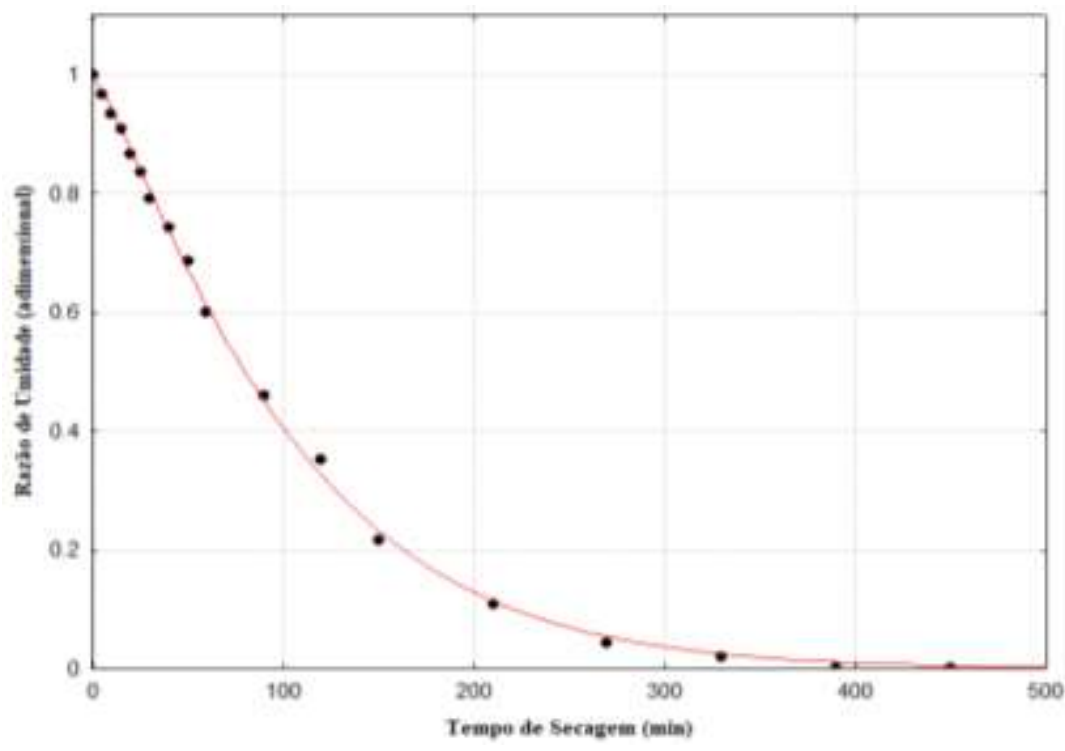

Fonte: Autores (2021). 
Figura 4. Ajuste do modelo de Logarítmico aos dados experimentais da secagem do resíduo de abacaxi.

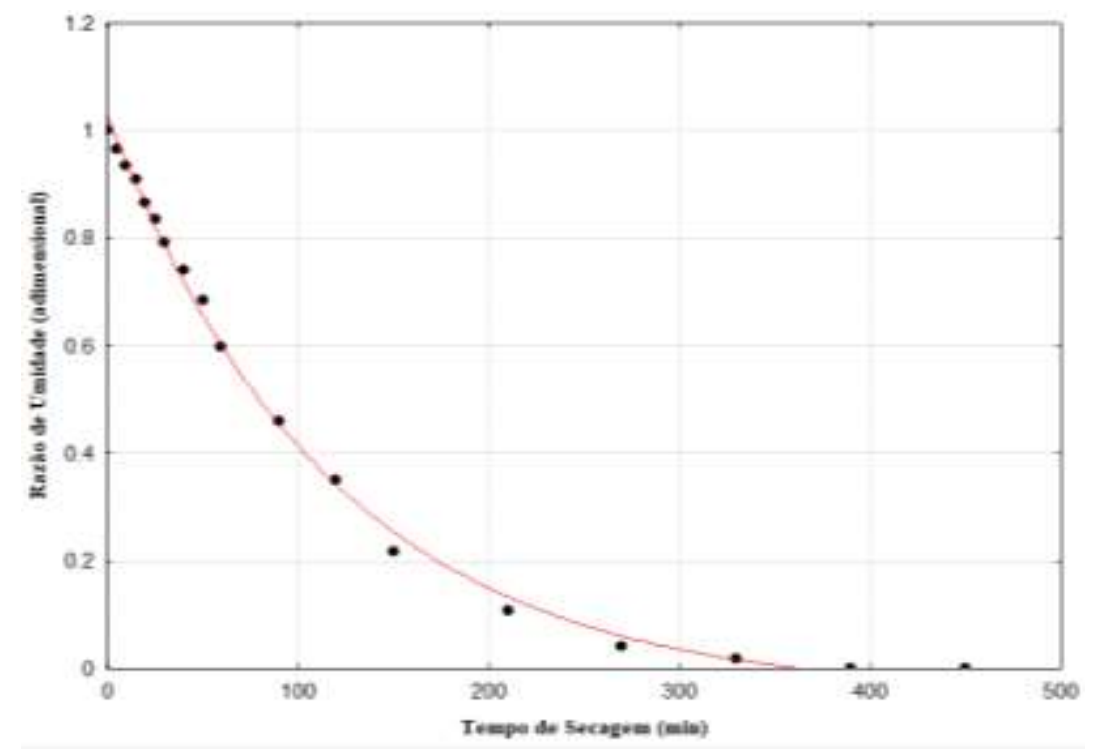

Fonte: Autores (2021).

Figura 5. Ajuste do modelo de Lewis aos dados experimentais da secagem do resíduo de abacaxi.

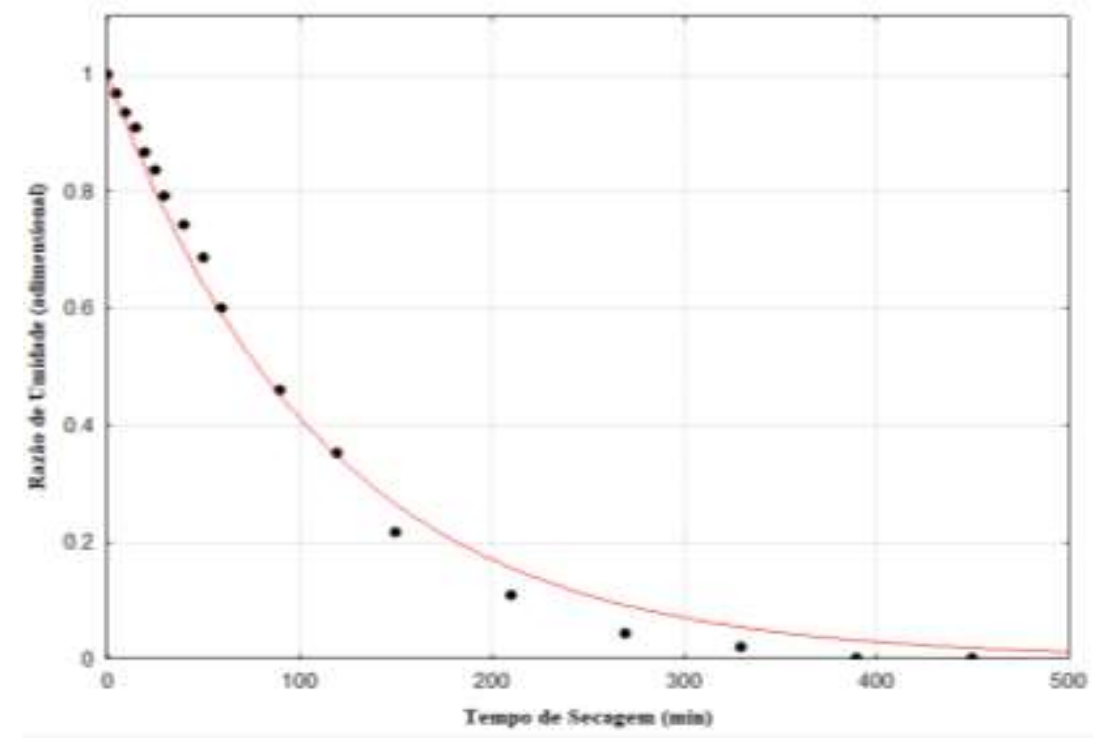

Fonte: Autores (2021).

Verifica-se nas figuras acima que os modelos testados no processo de secagem resultaram em bons ajustes aos dados experimentais, com uma melhor demonstração do fenômeno de secagem do resíduo de abacaxi devido as curvas ajustadas pelos modelos terem se aproximado dos resultados experimentais, assim confirmando o desempenho deles.

\subsection{Caracterização físico-química do pó do resíduo de abacaxi}

Para o pó foram determinados a umidade, $\mathrm{pH}$, acidez total titulável, sólidos totais e cinzas, os quais estão apresentados na Tabela 5 abaixo. 
Tabela 5. Parâmetros físico-químicos do pó do resíduo de abacaxi.

\begin{tabular}{cc}
\hline PARÂMETROS & RESULTADOS \\
\hline Umidade $(\%)$ & $4,27 \pm 0,05$ \\
\hline $\mathrm{pH}$ & $4,04 \pm 0,03$ \\
\hline Acidez Titulável Total (\%) & $4,92 \pm 0,11$ \\
\hline Sólidos Solúveis ( ${ }^{\circ}$ Brix) & $25,20 \pm 0,04$ \\
\hline Cinzas (\%) & $6,70 \pm 0,27$ \\
\hline
\end{tabular}

Fonte: Autores (2021).

O teor de umidade é uma das análises feitas em alimentos mais importante devido a sua relação com a estabilidade, qualidade e composição que pode afetar no seu processamento e armazenamento. Verifica-se na tabela 5 um valor de $4,27 \%$ no parâmetro de umidade evidenciando que o processo de secagem contribuiu significativamente para a redução no teor de água existente no resíduo de abacaxi. Lima (2019) encontrou um valor de 2,003\% de umidade sendo este inferior ao encontrado no presente estudo, porém ambos dentro do padrão estabelecido pela legislação brasileiro para farinhas de vegetais de no máximo 15\% de teor de água (Brasil, 1978). Dessa forma, a farinha obtida pode ser considerada um produto de fácil conservação devido apresentar um baixo teor de umidade.

Além da umidade, segundo Vilar et al. (2020) o pH é uma das propriedades mais importantes para avaliação de alimentos, devido indicar se os produtos são seguros em relação ao crescimento de microrganismos. O valor encontrado referente ao $\mathrm{pH}$ de 4,04 \pm 0,03 considerado ácido, pois foi inferior ao $\mathrm{pH}$ de segurança 4,5. Esses resultados são possíveis em virtude da grande quantidade de ácido cítrico e ácido ascórbico que existe na polpa (parte comestível), como também na casca da fruta (Lima, 2019).

A medida de acidez total titulável dos alimentos representa todos os grupamentos ácidos encontrados (ácidos orgânicos livres, na forma de sais e compostos fenólicos) (Fontes et al., 2005). O valor obtido para acidez titulável 4,92 $\pm 0,11$ sendo este superior ao encontrado por Vieira et al. (2019) de 2,13 \pm 0,01 (\% ácido cítrico) para a farinha mista de resíduo de abacaxi e caju. Com isso, segundo Fernandes et al. (2008) a farinha do resíduo de abacaxi pode ser classificada como produto ácido, apresentando a característica de reduzir a possibilidade de ataque microbiano, devido ao seu pH ácido ser considerado benéfico para conservação do alimento.

O teor de sólidos solúveis em ${ }^{\circ}$ Brix encontrado no presente trabalho foi de $25,2 \pm 0,04$. Estes valores são decorrentes da alta concentração de açúcares presente no miolo do abacaxi, o que confere um grau relativamente elevado de doçura à farinha. Resultados próximos ao presente trabalho foram relatados por Lemos e colaboradores (2010), os quais encontraram valores de 26,66 ${ }^{\circ}$ Brix em farinha contendo somente casca de abacaxi.

Quanto ao teor de cinzas pode-se observar um aumento significativo referente aos minerais totais após o processo de secagem do resíduo de abacaxi, encontrando um teor de 6,70 0,27\%, sendo esse superior ao encontrado por Barros et al. (2019) de $2,10 \pm 0,51 \%$ para a farinha do resíduo do abacaxi seca a $70{ }^{\circ} \mathrm{C}$, no entanto ambos resultados são aceitáveis tendo em vista que quanto menor o teor de água maior a quantidade de matéria inorgânica (Batista Sobrinho, 2014).

\subsection{Caracterização do conteúdo bioativo total e capacidade antioxidante do resíduo de in natura e do pó do resíduo de abacaxi.}

Os resultados obtidos para caraterização do conteúdo bioativo total e capacidade antioxidante do resíduo de abacaxi in natura do pó do resíduo estão apresentados na Tabela 6. 
Tabela 6. Capacidade antioxidante total (AOX) do resíduo agroindustrial de abacaxi in natura e submetido a secagem convectiva (pó), segundo os métodos de captura do radical livre - DPPH, redução do ferro - FRAP e conteúdo bioativo total (CBT).

\begin{tabular}{ccc}
\hline Análise & In natura & Pó \\
\hline CBT $^{\Psi}$ & $1421,77 \pm 0,89 \mathrm{~b}$ & $4000,85 \pm 0,97 \mathrm{a}$ \\
\hline DPPH & $2,06 \pm 0,82 \mathrm{~b}$ & $10,33 \pm 0,75 \mathrm{a}$ \\
\hline FRAP & $8,88 \pm 0,72 \mathrm{~b}$ & $42,28 \pm 0,45 \mathrm{a}$ \\
\hline
\end{tabular}

Nota: AOX DPPH- Atividade antioxidante expressa como equivalente ao Trolox em $\mathrm{mM} \mathrm{Kg}^{-1}\left(\mathrm{mM} \mathrm{TEAC} \mathrm{L}^{-1}\right)$. FRAPpoder antioxidante de redução de ferro expresso em milimols de $\mathrm{Fe}^{2+}\left(\mathrm{mM} \mathrm{Fe} \mathrm{Kg}^{2+}\right)$. $\Psi$ Conteúdo Bioativo Total medidos com Folin-Ciocalteu expresso como mg. $\mathrm{Kg}^{-1}$ equivalente ao ácido gálico. Médias seguidas da mesma letra na linha, não diferem entre si pelo teste de Tukey ao nível de 5\% de probabilidade. Fonte: Autores (2021).

Ao analisar os resultados da tabela 6, percebe-se que tanto a capacidade antioxidante total por DPPH e FRAP como conteúdo bioativo total obtiveram diferença significativa $(\mathrm{p}<0,05)$ com valores superiores para o pó demonstrando que a secagem convectiva a $60^{\circ} \mathrm{C}$ tem um bom desempenho na concentração de compostos bioativos.

O conteúdo bioativo total para o pó do resíduo do abacaxi apresentou 4000,85 mg/Kg valor menor ao de Souza et al., (2021) relatou para o pó da casca de abacaxi extraídos por ultrassom aliado ao etanol, 2036,80 mg/g. Enquanto, o resultado para capacidade antioxidante por DPPH encontrado foi de 0,01273 mM Trolox/g, próximo ao do presente estudo. Aquino et al., (2020) apontou para os extratos do pó de casca de sapoti em metanol 40\% o valor de 92,1 $\mu \mathrm{M}$ Trolox/g para DPPH.

\subsection{Conteúdo bioativo total e capacidade antioxidante das barras de cereais}

Os teores de conteúdo bioativo total e capacidade antioxidante das formulações da barra de cereal padrão e com adição do pó do resíduo agroindustrial de abacaxi obtido por secagem convectiva estão descritos na Tabela 7.

Tabela 7. Capacidade antioxidante total (AOX) das formulações da barra de cereal, padrão (BP), com adição do pó do resíduo agroindustrial de abacaxi B1 (2.5\%), B2 (3.5\%) e B3 (4.5\%), segundo os métodos de captura do radical livre - DPPH, redução do ferro - FRAP e conteúdo bioativo total (CBT).

$\begin{array}{lllll}\text { Análise } & \text { BP } & \text { B1 } & \text { B2 } & \text { B3 }\end{array}$

\begin{tabular}{ccccc}
\hline CBT $^{\Psi}$ & $1885,35 \pm 0,47 \mathrm{a}$ & $2231,90 \pm 0,59 \mathrm{a}$ & $1783,36 \pm 0,55 \mathrm{a}$ & $2038,21 \pm 0,35 \mathrm{a}$ \\
\hline DPPH & $3,79 \pm 0,28 \mathrm{~b}$ & $4,31 \pm 0,21 \mathrm{~b}$ & $4,42 \pm 0,17 \mathrm{~b}$ & $5,20 \pm 0,44 \mathrm{a}$ \\
\hline FRAP & $17,97 \pm 0,72 \mathrm{a}$ & $19,18 \pm 0,05 \mathrm{a}$ & $21,17 \pm 0,50 \mathrm{a}$ & $22,73 \pm 0,23 \mathrm{a}$
\end{tabular}

Nota: AOX DPPH- Atividade antioxidante expressa como equivalente ao Trolox em mM Kg${ }^{-1}\left(\mathrm{mM} \mathrm{TEAC} \mathrm{L}^{-1}\right)$. FRAP- poder antioxidante de redução de ferro expresso em milimols de $\mathrm{Fe} 2+\left(\mathrm{mM} \mathrm{Fe}^{2+} \mathrm{Kg}^{-1}\right)$. $\Psi$ Conteúdo Bioativo Total medidos com Folin-Ciocalteu expresso como mg. $\mathrm{Kg}^{-1}$ equivalente ao ácido gálico. Médias seguidas da mesma letra na linha, não diferem entre si pelo teste de Tukey ao nível de $5 \%$ de probabilidade. Fonte: Autores (2021).

De acordo com a Tabela 7, as diferentes formulações da barra de cereal não apresentaram resultados estatisticamente distintos $(\mathrm{p}<0,05)$ para as análises de conteúdo bioativo total e FRAP. Entretanto, DPPH foi o teste determinante. Nesse último, observa-se que a formulação B3 com 4,5\% de pó do resíduo agroindustrial de abacaxi foi a melhor, enquanto as demais não deferiram estatisticamente, evidenciando que proporções mais altas do pó do resíduo de abacaxi proporcionam atividades antioxidantes superiores. 
Machado (2018) apresentou resultados similares para CBT nas formulações de barras de cereal com casca de uva com $15 \%$ e $20 \%$ de pó de casca de uva grossa e fina, sendo esses entre 172,15 e 245,26 mg AGE/100g. Diferentemente desse estudo Andrade (2019) encontrou para atividade antioxidante por FRAP valores significativamente maiores $(\mathrm{p}<0,05)$ à medida que $\mathrm{o}$ percentual de resíduo de manga aumentava.

\subsection{Parâmetros de Cor e Firmeza}

Na Tabela 8 estão apresentados os resultados dos parâmetros instrumentais de firmeza e cor obtidos para as formulações das barras de cereais padrão e com adição do pó do resíduo agroindustrial de abacaxi por secagem convectiva.

Tabela 8. Firmeza e cor das formulações da barra de cereal, padrão (BP), com adição do pó do resíduo agroindustrial de abacaxi B1 $(2.5 \%)$, B2 $(3.5 \%)$ e B3 $(4.5 \%)$.

\begin{tabular}{|c|c|c|c|c|c|c|}
\hline \multicolumn{3}{|c|}{ Formulações } & BP & B1 & B2 & B3 \\
\hline \multirow{4}{*}{ Parâmetros } & \multicolumn{2}{|c|}{ Firmeza (N) } & $16,17 \pm 0,45 \mathrm{a}$ & $21,29 \pm 0,58 \mathrm{a}$ & $18,44 \pm 0,74 \mathrm{a}$ & $12,43 \pm 0,50 \mathrm{a}$ \\
\hline & \multirow{3}{*}{ Cor } & $\mathrm{L}^{*}$ & $52,14 \pm 0,27 \mathrm{a}$ & $55,23 \pm 0,70 \mathrm{a}$ & $54,72 \pm 0,62 \mathrm{a}$ & $53,28 \pm 0,93 a$ \\
\hline & & $a^{*}$ & $7,82 \pm 0,43 b$ & $9,35 \pm 0,29 a$ & $9,47 \pm 0,17 \mathrm{a}$ & $8,70 \pm 0,65 \mathrm{ab}$ \\
\hline & & $\mathrm{b}^{*}$ & $28,50 \pm 0,23 a$ & $31,74 \pm 0,52 a$ & $31,59 \pm 0,30 a$ & $29,38 \pm 0,79 a$ \\
\hline
\end{tabular}

Nota: Médias seguidas da mesma letra na linha, não diferem entre si pelo teste de Tukey ao nível de 5\% de probabilidade. Fonte: Autores (2021).

Os testes de compressão pelo texturômetro exercem força sobre o produto para causar uma fratura ou ruptura que simula a mastigação e quanto menor a pressão resultante do teste, consequentemente mais macia será a amostra (Chen; Engelen, 2012; Carvalho et al., 2015).

Observou-se que para firmeza instrumental, a força aplicada não deferiu significativamente ao nível de $5 \%$ nas formulações padrão e com adição do pó do resíduo agroindustrial de abacaxi, indicando que o percentual de pó adicionado não alterou esse parâmetro. No presente estudo os valores variaram entre 12,43 a 21,29 N, corroborando com Oliveira et al., (2021) que encontrou 9,46 a 28,24 $\mathrm{N}$ para barra de cereal com farinha de bagaço de azeitona. E ficou abaixo dos resultados relatados por Rodrigues et al., (2018) para barra de cereal com bagaço de caju, 26,64 a 35,71 N.

Todos os resultados dos parâmetros colorimétricos obtidos foram positivos, indicando tendência de cor mais para o vermelho e amarelo. Sendo que, entre $a^{*}$ e $b^{*}$, somente a coordenada $a^{*}$ apresentou diferença significativa ao nível $5 \%$ para as barras de cereais com pó em relação a padrão. O valor de L* foi de 52,14 a 55,23, apontando luminosidade mais clara em relação a barra de cereal a base de coproduto do pedúnculo de caju (Anacardium occidentale L) e soro de leite de Lima et al., (2021) que obteve 43,3 a 48,7 .

\subsection{Perfil de Compostos Fenólicos por Cromatografia Líquida de Alta Eficiência (CLAE)}

Os resultados obtidos para a análise do perfil de compostos fenólicos (g.100 g $\mathrm{g}^{-1}$ ) do resíduo de abacaxi in natura (IN) e secagem convectiva a $60^{\circ} \mathrm{C}(\mathrm{S})$ estão apresentados na Tabela 9.

Em suma, a identificação e quantificação dos compostos fenólicos foi superior para o resíduo submetido a secagem convectiva a $60^{\circ} \mathrm{C}$, já que é possível observar o triplo de total de fenólicos quantificados. Sendo, o flavanol catequina o principal composto detectado com 28,00 e 93,20 g. $100 \mathrm{~g}^{-1}$ para resíduo in natura e seco, respectivamente. 
Ainda em flavanóis, outros compostos como epigalocatequina galato, procianidina B1 e B2 foram encontrados em menor concentração. Para flavonóis, foram identificados o kaempferol-3-glicosídeo para ambos os tratamentos e quercetina 3glicosídeo para o pó da secagem. Em relação aos compostos não flavonoides nada foi detectado para a amostra do resíduo in natura, entretanto há a presença do estilbeno trans-Resveratrol e dos ácidos caftárico e caféico em baixas concentrações para o resíduo seco.

Tabela 9. Perfil de compostos fenólicos $\left(\mathrm{g} .100 \mathrm{~g}^{-1}\right)$ do resíduo de abacaxi in natura (IN) e submetido a secagem convectiva a $60^{\circ} \mathrm{C}$.

\begin{tabular}{|c|c|c|}
\hline AMOSTRAS & IN & $\mathbf{S}$ \\
\hline \multicolumn{3}{|l|}{ Flavanóis } \\
\hline$(+)$-Catequina & $28,00 \pm 1,77 \mathrm{~b}$ & $93,20 \pm 0,92 \mathrm{a}$ \\
\hline (-) -Epicatequina & ND & ND \\
\hline (-) -Epicatequina galato & ND & ND \\
\hline (-) -Epigalocatequina galato & $1,47 \pm 0,06 \mathrm{~d}$ & $4,87 \pm 0,15 \mathrm{a}$ \\
\hline Procianidina A2 & ND & ND \\
\hline Procianidina B1 & $0,97 \pm 0,06 \mathrm{c}$ & $0,90 \pm 0,21 \mathrm{~b}$ \\
\hline Procianidina B2 & $1,47 \pm 0,06 \mathrm{e}$ & $3,37 \pm 0,15 b$ \\
\hline \multicolumn{3}{|l|}{ Flavonóis } \\
\hline Kaempferol-3-glicosídeo & $0,37 \pm 0,15 \mathrm{c}$ & $0,60 \pm 0,10 \mathrm{c}$ \\
\hline Rutina & ND & ND \\
\hline Quercetina 3-glicosídeo & ND & $1,60 \pm 0,10 \mathrm{a}$ \\
\hline \multicolumn{3}{|l|}{ Estilbenos } \\
\hline Cis-Resveratrol & ND & ND \\
\hline trans-Resveratrol & ND & $1,20 \pm 0,10 \mathrm{a}$ \\
\hline \multicolumn{3}{|l|}{ Ácidos fenólicos } \\
\hline Ácido caftárico & ND & $2,80 \pm 0,20 \mathrm{a}$ \\
\hline Ácido caféico & ND & $0,50 \pm 0,10$ \\
\hline TFQ por HPLC & $32,51 \pm 0,42 \mathrm{e}$ & $107,54 \pm 0,88 \mathrm{c}$ \\
\hline
\end{tabular}

Nota: Médias seguidas da mesma letra na linha, não diferem entre si pelo teste de Tukey ao nível de 5\% de probabilidade. Os resultados são expressos como média \pm desvio padrão $(n=3)$. ND - Não detectado. (TFQ) Total de fenólicos Quantificados por HPLC. Fonte: Autores (2021).

O resíduo de abacaxi in natura e obtido por secagem à $60^{\circ} \mathrm{C}$ apresentou maiores resultados catequina em comparação ao resíduo agroindustrial do abacaxí de Ferreira et al., (2020) que encontrou 1,44 g.100 g-1 para in natura e 7,83 g.100 g-1 para secagem à $55^{\circ} \mathrm{C}$. Enquanto, o estudo com distintas variedades de mangas de Marcillo-Parra et al., (2021) não detectou a catequina.

O artigo de Del Juncal-Guzmán et al., (2021) mostrou que o composto galocatequina foi o mais abundante nas barras de abacaxi e que a bioacessibilidade dessas foram de $45 \%$. Sendo a bioacessibilidade o parâmetro que quantifica a capacidade de um componente estar presente no intestino humano após liberado da matriz do alimento (Vela Paredes, 2017). Em contraposição, Brito et. al., (2021) apontou que para a farinha da coroa do abacaxi os principais compostos fenólicos foram os ácidos clorogênico, p-cumárico, ferúlico e caféico. 


\section{Conclusão}

O resíduo agroindustrial de abacaxi apresentou características físico-químicas favoráveis e um potencial funcional, devido a presença de compostos bioativos e atividade antioxidante demonstrando a possibilidade do seu emprego como aditivos ou na incorporação em formulação de alimentos.

O modelo de Page foi considerado como o mais adequado para representar o fenômeno investigado no presente estudo, por apresentar maior valor do coeficiente de determinação e baixo valor do erro quadrático médio.

A adição do pó do resíduo de abacaxi obtido por secagem convectiva a $60^{\circ} \mathrm{C}$ influenciou significativamente no aumento da concentração dos compostos bioativos totais e atividade antioxidante nas formulações das barras de cereais em comparação com a barra padrão.

O total de fenólicos quantificados (TFQ) evidenciou que o processo de secagem concentrou os compostos encontrados, sendo o flavanol catequina o principal composto detectado com 28,00 e 93,20 g.100 g-1 para resíduo in natura e desidratado, respectivamente.

\section{Agradecimentos}

Ao Instituto Federal do Sertão Pernambucano (IF Sertão - PE), e todos que colaboram na realização desse trabalho.

\section{Referências}

Ade. K. D., Lal, E. A., \& Rathid, A. S. (2014). Developmentand Quality Evaluation of Pineapple Pomace And Wheat Bran Fortified Biscuits. International Journal of Research in Engineering \& Advanced Technology, 2(3).

AOAC. (2005). Methods-W. In: Horwitz (Ed.). Official Methods of Analysis of AOAC International. 18th Ed. AOAC International Gaithersburg, MD, USA.

Andrade, J. O. (2019). Aproveitamento do resíduo de manga no desenvolvimento de barra de cereal: atividade antioxidante in vitro e avaliação sensorial. Monografia (Curso de Graduação em Nutrição). Cuité: CES. 51 fl. http://dspace.sti.ufcg.edu.br.

Aquino, T. A. C., Gualberto, N. C., Narain, N., \& Santana, L. C. L. de A. (2020). Evaluation of bioactive compounds from Sapodilla (Manilkara zapota) peel and seeds obtained by ultrasound-assisted technique. Research, Society and Development, $9(8)$, e354985158. https://rsdjournal.org/index.php/rsd/article/view/5158.

Batista Sobrinho, I. S. (2014). Propriedades nutricionais e funcionais de resíduos de abacaxi, acerola e cajá oriundos da indústria produtoras de polpas. 2014. 166p. Dissertação (Mestrado em Ciências Ambientais) - Universidade Estadual do Sudoeste da Bahia, Itapetinga.

Bortolatto, J., \& Lora, J. (2008). Avaliação da composição centesimal do abacaxi (Ananas comosus (L.) Merril) liofilizado e in natura. Revista de Pesquisa e Extensão em Saúde, 4(1), 25-38.

Bortolotti, C. T.; Santos, K. G.; Francisquetti, M. C. C.; Duarte, C. R.; \& Barrozo, M. A. S. (2013). Hydrodynamic Study of a Mixture of West Indian Chery Residue and Soybean Grains in a Spouted Bed. The Canadian Journal of Chemical Engineering, 91, 1871-1880.

Brasil. (1978). Comissão Nacional de Normas e Padrões para Alimentos. Resolução n 12, de julho 1978. Brasília, DF.

Brito, T. B. N., Lima, L. R. S., Santos, M. C. B., Moreira, R. F. A., Cameron, L. C., Fai, A. E. C., \& Ferreira, M. S. L. (2021). Antimicrobial, antioxidant, volatile and phenolic profiles of cabbage-stalk and pineapple-crown flour revealed by GC-MS and UPLC-MSE. Food Chemistry. 339. https://www.sciencedirect.com/science/article/pii/S0308814620317441.

Brizola, R. \& Bampi, G. B. (2014). Desenvolvimento de barras alimentícias com adição de farinha de banana verde. Unoesc \& Ciência - ACBS, 5, 63-68.

Chen, J., \& Engelen, L. (2012). Food Oral Processing: Fundamentals of Eating and Sensory Perception. Chichester: Wiley-Blackwell. 408 p.

Del Juncal-Guzmán, D., Hernández-Maldonado, L. M., Sánchez-Burgos, J. A., González-Aguilar, G. A., Ruiz-Valdiviezo, V. M., Tovar, J., \& Sáyago-Ayerd, S. G. (2021). In vitro gastrointestinal digestion and colonic fermentation of phenolic compounds in UV-C irradiated pineapple (Ananas comosus) snack-bars. LWT. v. 138. https://www.sciencedirect.com/science/article/pii/S0023643820316248.

Ferreira, E. T. J., De Brito Araújo, A. J., \& Dos Santos Lima, M. (2020). Potencial funcional associado a compostos bioativos do resíduo agroindustrial da manga' palmer'. Jornada de Iniciação Científica e Extensão, 15(1). 66.

IAL. Instituto Adolfo Lutz. Normas analíticas do Instituto Adolfo Lutz: métodos químicos e físicos para análise de alimentos. (4a ed.), 1.020p. 2008.

Instituto Brasileiro de Geografia e Estatística. (2016). Sistema IBGE de Recuperação Automática. https://sidra.ibge.gov.br/tabela/1618.

Karovicová, Z. J., \& Magala, M. (2013). Rheological and qualitative characteristics of pea flour incorporated cracker biscuits. Croat. J. Food Sci. Technol. 5, 11-17. 
Kaveh, M., Chayjan, R. A., \& Nikbakht, A. M. (2017). Mass transfer characteristics of eggplant slices during length of continuous band dryer. Heat and Mas $s$ Transfer, 53(6), 2045-2059.

Kim, Y. K., Guo, Q., \& Packer, L. (2002). Free radical scavenging activity of red ginseng aqueous extracts. Toxicology, 172, $149-156$.

Lima, A. B. M. (2019). Obtenção de farinha de abacaxi a partir da secagem dos resíduos agroindústriais da fruta para aplicação em formulações de cookies. Trabalho de conclusão, UFRN - Rio Grande do Norte.

Lima, S. K. R., Nóbrega, M. M. G. P., Carneiro, R. M., Silva, R. A. da ., Medeiros, S. R. A., \& Muratori, M. C. S. (2021). Formulation of food bars based on the co-product of cashew peduncle (Anacardium occidentale L) and whey. Research, Society and Development, $10(1), \mathrm{e} 7010111213$.

Macagnan, F. T., Moura, F. A., Santos, L. R., Bizzani, M., \& Silva, L. P. (2014). Caracterização nutricional e resposta sensorial de pães de mel com alto teor de fibra alimentar elaborados com farinhas de subprodutos do processamento de frutas. Boletim do Centro de Pesquisa de Processamento de Alimentos, 32.

Machado, A. M. R. (2018). Utilização de casca de uva como ingrediente no desenvolvimento de barras de cereais. Dissertação (Mestrado em Ciência de Alimentos) - Instituto de Química, Universidade Federal do Rio de Janeiro.

Marcillo-Parra, V., Anaguano, M., Molina, M., Tupuna-Yerovi, D. S., \& Ruales, J. (2021). Characterization and quantification of bioactive compounds and antioxidant activity in three different varieties of mango (Mangifera indica L.) peel from the Ecuadorian region using HPLC-UV/VIS and UPLC-PDA. NFS Journal. v. 23.

Martins, E. A. S., Lage, E. Z., Goneli, A. L. D., Hartmann Filho, C. P., \& Lopes, J. G. (2015). Cinética de secagem de folhas de timbó (Serjania marginata Casar). Revista Brasileira de Engenharia Agrícola e Ambiental, 19(3), 238-244. 10.1590/1807 1929/agriambi. v19n3p238-244.

Matos, A. T. (2014). Tratamento e Aproveitamento Agrícola de Resíduos Sólidos. Ed. UFV.

Menon, L., Majumdar, S. D., \& Ravi, U. (2014). Mango (Mangifera indica L.) kernel flour as a potential ingredient in the development of composite flour bread. Indian Journal of Natural Products and Resources. 5, 75-82.

Monteiro, L. F. et al. (2014). Secagem combinada convectivo-solar de resíduo enriquecido de casca de abacaxi. In: XX Congresso Brasileiro de Engenharia Química-COBEQ, 2014. Anais... Blucher, 3277-3284.

Moreno, J. de S. Obtenção, caracterização e aplicação de farinha de resíduos de frutas em cookies. (2016). 82 f. Dissertação (Mestrado) - Curso de Ciência de Alimentos, Universidade Estadual do Sudoeste da Bahia, Itapetinga.

Morzelle, M. C., Bachiega, P., Souza, E. C., Vilas Boas, E. V. B., \& Lamounier, M. L. (2015). Caracterização química e física de frutos de curriola, gabiroba e murici provenientes do cerrado brasileiro. Revista Brasileira de Fruticultura, 37, 96- 103.

Moscon, E. S., Martin, S., Spehar, C. R., Devilla, I. A., \& Rodolfo Junior, R. (2017). Cinética de secagem de grãos de quinoa (chenopodium quinoa w.). Revista Engenharia na Agricultura, 25(4), 318-325.

Nunes, J.S., Lins, A. D. F., Gomes, J. P., Silva, W. P., \& Silva, F. B. (2017). Influência da temperatura de secagem nas propriedades físico-química de resíduos abacaxi. Revista Agropecuária Técnica, 1(1), 41-46.

Nogueira, G. D. R. et al. (2019). Food and Bioproducts Processing Analysis of a hybrid packed bed dryer assisted by infrared radiation for processing acerola ( Malpighia emarginata D . C .) residue. Food and Bioproducts Processing, 114, 235-244.

Oliveira, A. A. N. (2018). Avaliação da Oxidação Lipídica em Hambúrguer de Carne Bovina Adicionado de Farinha da Casca do Abcaxi (Ananas comosus (L.) Merril) como Antioxidante Natural. Dissertação de Mestrado.

Oliveira, E. S. F., Soares, J. O., Silva, M. S., Orqis, M. G., Azevedo, M. L., \& Gautério, F. A. G. (2020). Perfil de textura de barras de cereal elaboradas com farinha de bagaço de azeitona contendo xantana e guar. $7^{\circ}$ Simpósio de Segurança Alimentar: Inovação com sustentabilidade.

Padilha, C. V. S., Miskinis, G. A., Souza, M. E. A. O., Pereira, G. E., Oliveira, D., Bordignon-Luiz, M. T., \& Lima, M. D. S. (2017). Rapid determination of flavonoids and phenolic acids in grape juices and wines by RP-HPLC/DAD: Method validation and characterization of commercial products of the new Brazilian varieties of grape. Food Chemistry, 228, 106-115.

Pereira, A. S., Shitsuka, D. M., Parreira, F. J., \& Shitsuka, R. (2018). Metodologia da Pesquisa Cientifica. UFSM.

Rodrigues, L. M. S., Alves, J. I. S., Almeida, R. D., Sousa, S., \& Mata, M. E. R. M. C. (2018). Perfil de textura instrumental de barras de cereais elaboradas com bagaço de caju. Congresso Técnico Científico da Engenharia e da Agronomia. Maceió-Al.

Rufino, M. D. S. M., Alves, R. E., Brito, E. S. DE., Morais, S. M. DE., Sampaio, C. D. G., Jiméneze, J. P., \& Calixto, F. D. S. (2006). Metodologia científica: determinação da atividade antioxidante total em frutas pelo método de redução do ferro (FRAP). Comunicado Técnico 125, 1-4.

Singleton, V. L., \& Rossi, J. A. Colorimetry of total phenolics with phosphomolybdic phosphotungstic acid reagents. (1965). American Journal of Enology and Viticulture, 16, 144-158.

Souza, M. E. A. O. de., Gomes, M. da R., Candeias, V. M. S., Albuquerque Junior, N. de M., Januário, E. T. F., Lima, D. A., \& Vilar, S. B. de O. (2021). Determination of the antioxidant capacity of pineapple peel powder extract by applying different extraction techniques. Research, Society and Development, 10(10), e155101018574.

Vela Paredes, R. S. Bioacessibilidade de compostos fenólicos e minerais em feijão comum (Phaseolus vulgaris L.). (2017). Escola Superior de Agricultura “Luiz de Queiroz". Dissertação (mestrado). USP. https://teses.usp.br/teses/disponiveis/11/11141/tde-20032018-133519/pt-br.php.

Vilar, S. B. O., Silva, J. N., Oliveira, I. P., Albuquerque, N. M. J., \& Coelho, A. B. (2020). Isoterma de Sorção de uva arra 15® desidratada e armazenada em diferentes embalagens. Congresso internacional da Agroindústria. 